\title{
Preface
}

\section{Unlocking Secrets of the Cancer Genome}

\author{
Jeffrey E. Green \\ National Cancer Institute, Bethesda, MD, USA
}

This issue of Breast Disease is dedicated to the emerging science using high-throughput genomic approaches to study breast cancer. These technologies offer great potential for stratifying the classification and treatment of patients based upon individual molecular profiles as well as identifying important new targets for therapy. The application of gene expression profiling to breast cancer research has already revolutionized clinical oncology and is being vigorously applied by the pharmaceutical industry for translational research.

The contributors to this issue of Breast Disease have made important advances in the field of breast cancer research from both the basic science and clinical oncology perspectives. Genomic approaches based upon single nucleotide polymorphisms (SNPs) are being used to identify genetic loci that contribute to breast cancer development and progression (see Struewing). Important molecular distinctions between a large panel of cancer cell lines have been identified using expression profiling and the identified gene clusters may be related to the biologic responses of the cell lines to a panel of over 100,000 screened compounds (see Weinstein). This serves as a proof-of-principle that such comprehensive and integrated genomic approaches can be successfully utilized. These types of approaches to cancer and pharmacogenomics will greatly accelerate drug discovery and individualized therapy.

Key advances have already been made regarding an expanded molecular classification of human breast cancer that transcends traditional tumor marker approaches. Clusters of genes that appear to be fingerprints for the dysregulation of specific pathways are being identified (such as for BRCA1 and BRCA2 mutations and ER status) and may be powerful tools for determining the natural history of a patient's tumor and appropriate therapeutic regimes (see reviews by Miller, Fuqua, and Meltzer). Efforts are currently underway to translate this work into new diagnostic methods for characterizing breast cancer.

While these clinical advances are truly spectacular, much remains to be learned about the molecular events involved in the etiology and progression of breast cancer. Ironically, it may be the little mouse that will continue to open doors into our understanding of breast cancer, ultimately leading to new targets for prevention and therapy. For this reason, half of the reviews in this issue are devoted to demonstrating the utility and contributions of mouse models for studying mammary cancer, especially through the application of new genomic technologies. The mouse offers an important means for understanding the molecular biology of mammary gland development and differentiation, processes that appear intimately linked to oncogenesis. Expression profiling has revealed changes in genes expression which occur during normal mammary gland development and differentiation (see Master) providing a basis for comparison to genes dysregulated during tumor formation (see Ye).

In certain models, dynamic changes in the organization of the mouse genome during mammary cancer progression may recapitulate very similar changes that occur during human breast cancer evolution (see Ried). The recent application of array CGH to studying genome evolution will undoubtedly lead to the identification of additional genes involved in breast cancer progression. Unlike the genetically diverse human population, the genetic background of mouse models can be selected, studied and compared to determine the strain influences on disease manifestations. Many studies have taken advantage of mouse genetics to identify 
susceptibility loci and recently this has been applied to localizing modifiers of mammary cancer metastases in mice (see Hunter).

Ultimately, there will be a better convergence of information derived through basic science approaches using animal models and human breast cancer. As ge- nomic technologies produce a flood of information regarding breast cancer, the great challenge will remain to use ever improving informatics approaches to identify the genes and networks which must be understood to prevent and cure this hideous disease. 